\title{
Essai d'isolement de Campylobacter dans la viande de volaille en République du Bénin
}

\author{
H.S. BANKOLE ${ }^{1,3}$, F. BABA-MOUSSA ${ }^{2}$, J.A. AGBANKPE ${ }^{3}$, T.V. DOUGNON ${ }^{1 *}$, \\ M. LEGONOU ${ }^{3}$, F. TOUKOUROU ${ }^{2}$ et L. BABA-MOUSSA ${ }^{4}$ \\ ${ }^{I}$ Ecole Polytechnique d'Abomey-Calavi, Laboratoire de Recherche en Biologie Appliquée, Université \\ d'Abomey-Calavi, 01 BP 2009 Cotonou, Bénin. \\ ${ }^{2}$ Laboratoire de Microbiologie et de Technologie Alimentaire, Faculté des Sciences et \\ Techniques/Université d'Abomey-Calavi, ISBA-Champ de foire Cotonou, Bénin. \\ ${ }^{3}$ Laboratoire de Microbiologie Alimentaire, Ministère de la Santé, 01 BP 418 Cotonou, Bénin. \\ ${ }^{4}$ Laboratoire de Biologie et de Typage Moléculaire en Microbiologie, Faculté des Sciences et \\ Techniques/Université d'Abomey-Calavi, 05 BP 1604 Cotonou, Bénin. \\ *Corresponding author; E-mail: victorien88@hotmail.com; Tel.00 (229) 97736446.
}

\section{RESUME}

La viande de volaille importée occupe une place importante dans l'alimentation béninoise. Elle est le plus souvent mise en cause dans les cas de toxi-infections alimentaires. De ce fait, elle devrait être sujette à un contrôle de tous les microorganismes de toxi-infections afin de préserver la santé des consommateurs. Mais il s'avère que Campylobacter, qui de plus en plus est indexé, ne fait pas partie de la gamme des bactéries recherchées pour la qualification sanitaire des aliments au Bénin. Cette situation serait due aux probables difficultés liées à l'isolement de ce germe. La présente étude a essayé d'isoler par culture cette bactérie afin de prouver que sa recherche en microbiologie alimentaire est possible. Cela pourrait permettre de commencer par l'inclure dans les diagnostics ultérieurs. Pour ce faire, 30 échantillons de viande de volaille importée ont fait l'objet d'analyse au Laboratoire de Microbiologie du Ministère de la Santé.

Sur les 30 échantillons, Campylobacter a été isolé dans 6, soit un taux de contamination de 20\%. En somme, Campylobacter peut être isolé par culture dans notre contexte.

(c) 2012 International Formulae Group. All rights reserved.

Mots clés : Campylobacter, viande de volaille, maladies alimentaires, Bénin.

\section{INTRODUCTION}

La qualité microbiologique des aliments constitue aujourd'hui une préoccupation majeure pour toute la chaîne agroalimentaire, de la matière première au produit fini. Les pays du Nord très tôt ont compris l'importance du contrôle microbiologique des produits de consommation et de l'implication des microorganismes dans la survenue des toxiinfections alimentaires. L'Organisation Mondiale de la Santé rapporte que des centaines de millions de personnes dans le monde souffrent de maladies dues à la contamination des aliments et que les denrées d'origine animales viennent en tête des causes identifiées (Moussiliou, 2008). Les toxiinfections d'origine alimentaire en particulier 
celles imputables à Campylobacter constituent une des causes les plus fréquentes de maladies intestinales, d'origine bactérienne chez l'homme (Puterflam et al., 2007). Le nombre déclaré de campylobactériose est en augmentation dans de nombreux pays européens (Martine et al., 2007). En France, le nombre annuel de cas d'infections à Campylobacter est estimé à environ 500000 (Martine et al., 2007). Des niveaux semblables de cas peuvent être détectés dans d'autres parties de l'Europe. Aux États-Unis, environ 2,4 millions de cas de Campylobacter surviennent chaque année (Kaakoush et al., 2011). L'infection à Campylobacter, se manifeste particulièrement par des diarrhées, des douleurs abdominales, des selles sanguinolentes, de la fièvre et parfois des nausées et des vomissements. Des complications peuvent s'observer. Au nombre de ces complications on peut noter, la septicémie, la méningite et le syndrome de Guillain-Barré (Kaakoush et al., 2011) ; ce qui dénote l'intérêt pour ces infections en santé publique. La viande de volaille, constitue un réservoir régulier de Campylobacter (Puterflam et al., 2007). La bactérie colonise le tractus gastro-intestinal de l'animal et se retrouve dans la viande crue (AFSSA, 2004).

La volaille est une denrée alimentaire problématique en raison de la concentration des abattages et de l'augmentation des élevages en plein air (Régula, 2008). La bactérie est transmise à l'homme, soit par un contact avec l'animal infecté, soit par ingestion d'aliments contaminés (AFSSA, 2004).

Au Bénin, l'importation de la viande de volaille a connu une augmentation d'environ $98 \%$ de 1999 à 2003. Ces importations viennent des pays où des cas de toxiinfections alimentaires à Campylobacter sont régulièrement notés. Paradoxalement,
Campylobacter ne fait pas partie de la liste des microorganismes recherchés dans le cadre de la qualification sanitaire de la viande de volaille dans notre pays. C'est pour parer à cette situation que le présent travail a été initié. L'objectif principal est d'évaluer le risque de toxi-infection alimentaire à Campylobacter due à la consommation de la viande de volaille importée au Bénin.

\section{MATERIEL ET METHODES}

Matériel

Dans le cadre de la présente étude, 30 échantillons de viande de volaille (ailerons de dindes, cuisses de poules et poules entières) obtenus au port autonome de Cotonou ont été utilisés. Le matériel utilisé est composé d'équipements de laboratoire pour usage microbiologique, de réactifs et de milieux de culture.

\section{Préparation des milieux de culture}

Tous les milieux de culture utilisés ont été préparés et stérilisés conformément aux instructions des fabricants.

\section{Préparation des suppléments Supplément Preston Campylobacter Technique}

Deux ml d'eau distillée stérile sont ajoutés au supplément Preston Campylobacter (Oxoid CM, England) dans les conditions aseptiques. Après homogénéisation par agitation jusqu'à dissolution complète, le contenu du flacon est transféré dans $500 \mathrm{ml}$ de gélose Campylobacter Preston.

\section{Supplément Karmali Campylobacter \\ Technique}

Après avoir ouvert sous flamme du bec Bunsen un flacon du supplément Karmali Campylobacter (IVD 81036), il faut $y$ introduire $2 \mathrm{ml}$ d'eau distillée stérile à l'aide d'une pipette graduée. Après homogénéisation 
par agitation jusqu'à dissolution complète, il faut renverser le contenu du flacon dans 500 ml de gélose Karmali, Campylobacter.

\section{Prélèvement}

Trois types de viande de volaille importée ont été prélevés à savoir, ailerons de dindes, cuisses de poules, poules entières. Pour chaque type de viande, nous avons sélectionné au hasard 5 conteneurs de différents pays. Les échantillons ont été prélevés dans des sachets stériles de Stomacher puis transportés au laboratoire dans des glacières contenant des accumulateurs. Au total, 30 échantillons ont été prélevés dans 15 conteneurs. Les Tableaux 1 et 2 montrent la répartition de ces échantillons selon les différents types de viande de volaille et leur provenance.

\section{Analyses microbiologiques}

$\begin{array}{llll}\begin{array}{l}\text { Recherche de Campylobacter } \\ \text { enrichissement et isolement }\end{array} & \text { par } \\ \text { Pour l'enrichissement, } & 25 & \mathrm{~g}\end{array}$
d'échantillon ont été introduits dans un flacon stérile contenant $250 \mathrm{ml}$ de bouillon Preston (ISO 10272-1:1995) enrichi au sang frais de mouton et au supplément Preston (CM0689), puis l'ensemble obtenu a été incubé à $42{ }^{\circ} \mathrm{C}$ pendant 48 heures.

\section{Isolement}

Les boîtes pré-coulées de Preston et de Kamali ont été ensemencées par stries avec les subcultures de $48 \mathrm{~h}$ puis disposées dans une jarre anaérobiose et incubées à l'étuve à $42{ }^{\circ} \mathrm{C}$ pendant $36 \mathrm{~h}$.

\section{Confirmation}

Le frottis frais et la coloration de Gram ont été réalisés pour confirmer le diagnostic (Corry et al., 2003). Les tests de catalase et de l'oxydase ont été faits conformément à la méthode décrite par Cowan et Steel (1974).

\section{Analyses statistiques}

Les données obtenues ont été analysées au moyen des logiciels Excel et MINITAB. Le test $\mathrm{t}$ de Student a été appliqué à un seuil de signification de 5\%. Les fréquences ont été calculées.

\section{RESULTATS}

Les sources de provenance de la viande de volaille sont très diversifiées (11 pays) (Tableau 1). Le poulet et la dinde sont les types de viande de volaille importés en République du Bénin (Tableau 2). Sur la gélose Preston agar, les colonies de Campylobacter sont légèrement roses, rondes et brillantes, avec un bord régulier (Figure 1). Pour la gélose Karmali agar, les colonies de Campylobacter sont grisâtres, plates et humides présentant un reflet métallique et ayant une tendance à l'étalement (Figure 2). L'observation microscopique a montré que, à l'état frais, Campylobacter présente une mobilité en vrille. Les cultures plus âgées sont moins mobiles. Avec la coloration de Gram, il se présente sous forme de bacilles à Gram négatif, incurvés ou spiralés (Figure 3a). Les vieilles cultures présentent des formes coccobacillaires. Les observations sont identiques à celles réalisées au niveau de la souche de référence (Figure $3 b$ ).

Sur les 30 échantillons de viande de volaille analysés, 6 étaient contaminés par Campylobacter spp, soit un taux de contamination de $20 \%$ (Tableau 3). La contamination par Campylobacter a été observée avec la viande de volaille en provenance du Brésil, de l'Espagne et de l'Italie (Tableau 4). 
Tableau 1 : Répartition des échantillons de viande de volaille selon la provenance.

\begin{tabular}{lll}
\hline Nature & Provenance & Effectifs \\
\hline Ailerons de dindes & France & 4 \\
Ailerons de dindes & Pologne & 2 \\
Ailerons de dindes & Hollande & 2 \\
Ailerons de dindes & Italie & 2 \\
Cuisses de poules & Brésil & 6 \\
Cuisses de poules & Hollande & 2 \\
Cuisses de poules & Grèce & 2 \\
Poules entières & Espagne & 2 \\
Poules entières & Argentine & 4 \\
Poules entières & Grande - Bretagne & 2 \\
Poules entières & Allemagne & 2 \\
Total & & $\mathbf{3 0}$ \\
\hline
\end{tabular}

Tableau 2 : Répartition des échantillons de viande de volaille selon la nature des différents types de viande de volaille.

\begin{tabular}{lc}
\hline Nature & Effectif \\
\hline Ailerons de dindes & 10 \\
& $(33,33 \%)$ \\
\hline Cuisses de poules & 10 \\
& $(33,33 \%)$ \\
\hline Poules entières & 10 \\
& $(33,33 \%)$ \\
\hline Total & $\mathbf{3 0}$ \\
\end{tabular}

Tableau 3 : Répartition des échantillons de viande de volaille en fonction du résultat de la recherche de Campylobacter.

\begin{tabular}{lccc}
\hline Nature & \multicolumn{2}{c}{ Résultats } & \multirow{2}{c}{$\begin{array}{c}\text { Effectifs } \\
\mathbf{( 3 0})\end{array}$} \\
\cline { 2 - 3 } & + & - & \\
\hline Ailerons de dindes & $(6)$ & $(24)$ & \\
& $(10 \%)$ & $(90 \%)$ & $(100 \%)$ \\
\hline Cuisses de poules & 3 & 7 & 10 \\
& $(30 \%)$ & $(70 \%)$ & $(100 \%)$ \\
\hline Poules entières & 2 & 8 & 10 \\
& $(20 \%)$ & $(80 \%)$ & $(100 \%)$ \\
\hline
\end{tabular}


Tableau 4 : Taux de contamination des échantillons par Campylobacter selon la provenance.

\begin{tabular}{lcc}
\hline Nature d'échantillon & Provenance & Taux de contamination \\
\hline Ailerons de dindes & Italie & $50 \%(1 / 2)$ \\
Cuisses de poules & Brésil & $50 \%(3 / 6)$ \\
Poules entières & Espagne & $100 \%(2 / 2)$ \\
\hline
\end{tabular}

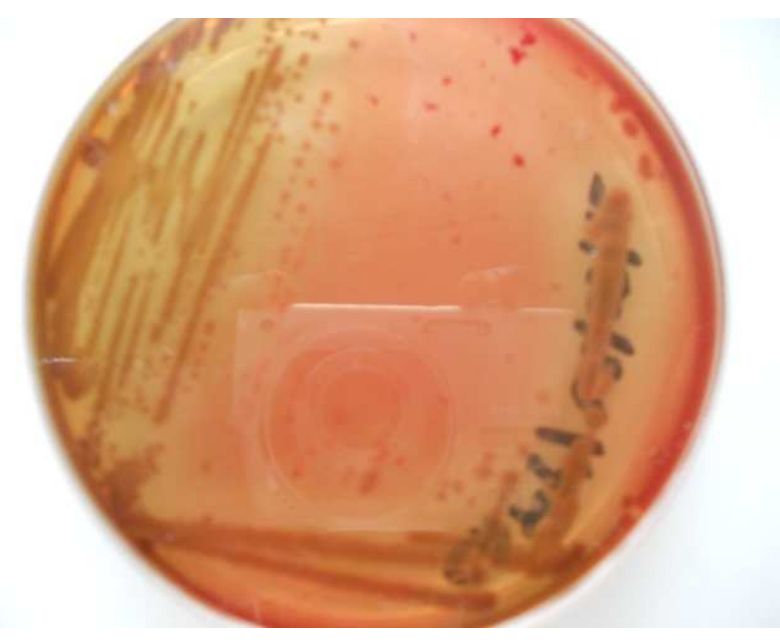

Figure 1 : Plaque de Preston agar portant des colonies de Campylobacter spp provenant de l'un des échantillons de viande de volaille.

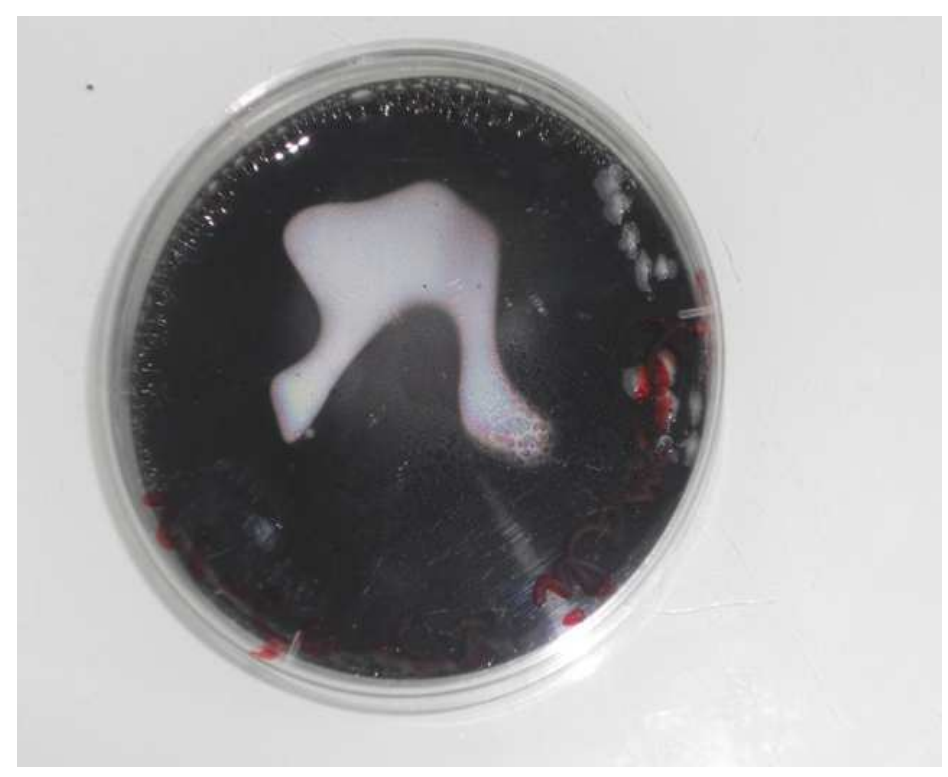

Figure 2 : Plaque de Karmali agar portant des colonies de Campylobacter spp provenant de l'un des échantillons de viande de volaille. 


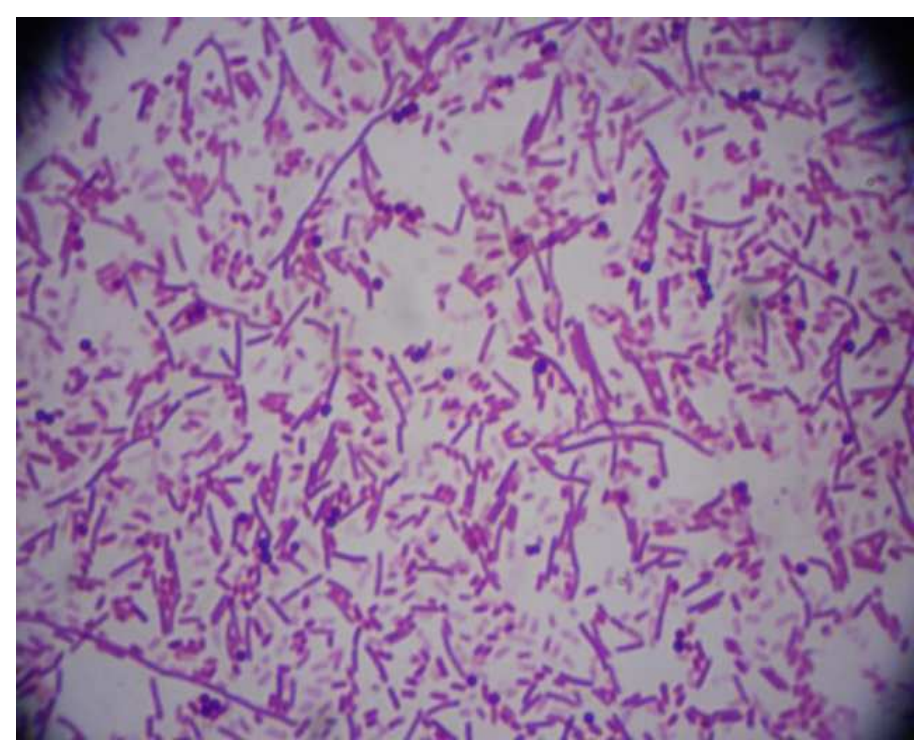

Figure 3a : Frottis réalisé à partir de la souche de référence.

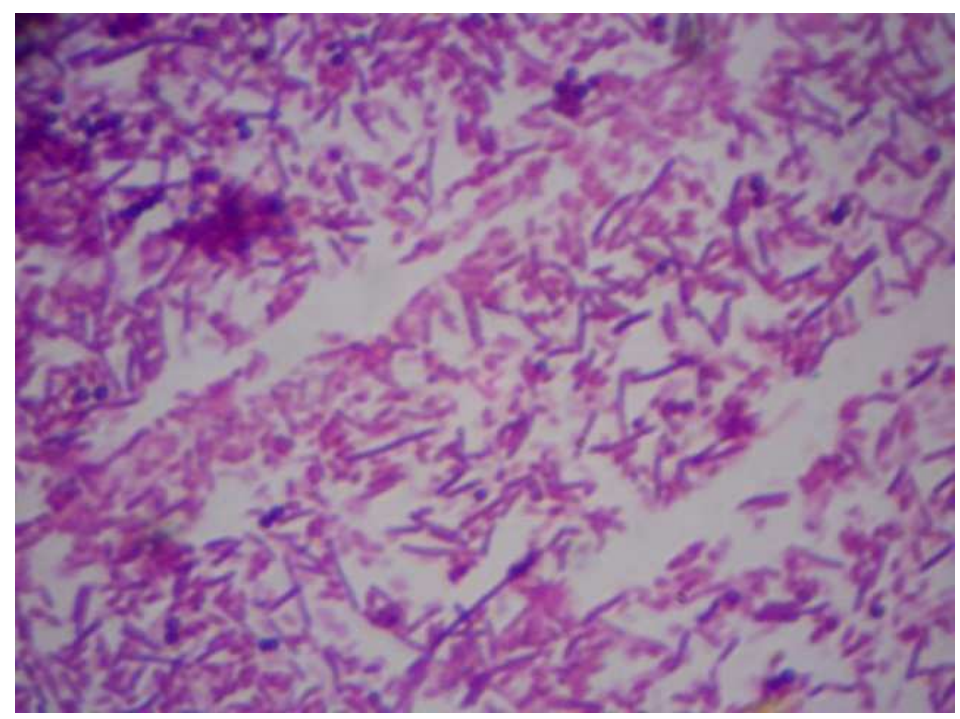

Figure 3b: Frottis réalisé à partir de la souche d'un échantillon de viande de volaille.

\section{DISCUSSION}

Flore d'intérêt nouveau au Bénin compte tenu de ses caractéristiques de culture, Campylobacter a été détecté dans six échantillons de viande de volaille sur les trente échantillons analysés; soit un taux de contamination de $20 \%$. Au regard de ce taux de contamination $(20 \%)$, on peut dire qu'un risque potentiel de toxi-infection à Campylobacter existe chez les consommateurs de viande de volaille mal cuite. Toutefois le taux de contamination obtenu, est inférieur à 
celui trouvé en Grande-Bretagne, par Puterflam et al. (2007) (29\%). Ils avaient utilisé des produits issus de l'abattage des poulets de chair. Ces résultats montrent bien que ce risque potentiel de toxi-infection à Campylobacter, n'est pas le fait de notre pays mais que la contamination observée, s'est réalisée depuis les pays exportateurs. Cette situation pourrait découler du non-respect du principe HACCP lors de l'abattage des volailles et lors de la transformation en produits de coupe (ailerons de dindes, cuisses de poules, etc.).

Des taux plus élevés de contamination de la viande de volaille ont été obtenus dans certains pays africains. C'est le cas du Sénégal où Cardinal en 2005, a obtenu un taux de $52 \%$. C'est aussi le cas de la Tunisie en 2000, où Dilmi et Belhadri a eu $65 \%$ des échantillons de viande de volaille contaminée par Campylobacter. Ces forts taux pourraient être imputables à l'hygiène et à la salubrité des manipulations lors des opérations de transformations. En effet, ces pays réalisent sur place la transformation.

Tous ces résultats ne font que confirmer que le risque d'infection à Campylobacter reste permanent chez les consommateurs de la viande de volaille.

Les tests de confirmations qui ont été réalisés sur les souches isolées de Campylobacter sont conformes à ceux relevés des travaux de Aspinall et al. (1993) et Corry et al. (2003).

Les différents types de viande de volaille analysée à savoir, ailerons de dindes, cuisses de poules, poules entières étaient respectivement contaminés par Campylobacter dans les pourcentages de $10 \%, 30 \%$ et $20 \%$ (Tableau 4). La comparaison de la contamination selon les différents types de viande de volaille au moyen du Kruskal-Wallis test a montré qu'il n'y a aucune différence statistiquement significative entre les produits du point de vue de la contamination aux Campylobacter ( $\mathrm{p}>$ $0,05)$. Ce qui montre que la contamination des viandes de volailles par Campylobacter ne dépend pas du type de viande de volaille.

Notre étude a montré que la totalité des échantillons provenant de l'Espagne a été contaminée par Campylobacter (Tableau 4), de même $75 \%$ des cuisses de poules provenant du Brésil ont été contaminés par Salmonelles (Tableau 4). La comparaison statistique de la contamination des viandes de volailles selon le pays d'origine montre qu'il y a une différence statistiquement significative entre les produits et leur pays d'origine $(\mathrm{p}<0,05)$. On peut donc dire que la contamination aux Campylobacter et aux Salmonelles des viandes de volailles dépend de la provenance de ces produits. Ce qui montre que le principe HACCP depuis la matière jusqu'au produit fini (cuisses de poules, ailerons de dindes, etc.) n'est pas bien suivi dans ces pays.

\section{Conclusion}

Sur les trente échantillons de viande de volaille utilisés pour la présente étude, Campylobacter a été isolé par culture dans six. Ce qui donne un taux de contamination de $20 \%$. Cette situation est la preuve qu'il existe un risque potentiel de toxi-infection à Campylobacter chez les consommateurs de la viande de volaille importée. Les Responsables ayant en charge la qualification sanitaire des aliments doivent alors prendre en compte Campylobacter dans tous les contrôles.

\section{REFERENCES}

AFSSA (Agence Française de Sécurité Sanitaire des Aliments). 2004. Appréciation des risques alimentaires liés aux Campylobacter: Application au couple poulet/Campylobacter jejuni. AFSSA ; 96p.

Aspinall S, Wareing D, Hayward P, Hutchinson D. 1993. Selective medium for thermophilic Campylobacters including Campylobacter upsaliensis. J. Clin. Pathol., 46: 829-831. 
Cardinal E. 2005. Salmonella et Campylobacter dans la filière avicole sénégalaise : impact sur la santé publique. Thèse de doctorat, Université de Dakar, Sénégal, p. 156.

Corry J, Atabay H, Forsythe S, Mansfield L. 2003. Culture media for the isolation of Campylobacters, Helicobacter and Arcobacters. In Handbook of Culture Media for Food Microbiology (2nd edn). Elsevier: Amsterdam, The Netherlands; 271-315.

Cowan ST, Steel KJ. 1974. Manual for the Identification of Medical Bacteria (2nd edn). Cambridge University Press: London; $45-122$.

Dilmi B, Belhadri A. 2000. Etude de la prévalence d'Hélicobacter pylori et de Campylobacter jejuni dans les viandes de volailles et les risques d'infection. Université Hassiba Ben Bouali - Chlef; Maroc, p. 5.

Kaakoush N, Deshpande P, Wilkins M, Raftery J, Janitz K, Mitchell H. 2011.
Comparative analyses of Campylobacter concisusstrains reveal the genome of the reference strain BAA-1457 is not representative of the species. Gut. Pathog., $3: 15$.

Martine D, Berengere C, Francis M, Philippe F. 2007. Caractérisation génétique d'isolats de Campylobacter récoltés en Bretagne et issus de différentes sources. Bulletin Epidémiologique, 30: 1-5.

Moussiliou A. 2008. Mise au point d'une méthode d'identification des Salmonella dans les denrées alimentaires. Mémoire de maîtrise professionnelle en Biotechnologie dans les Industries Agroalimentaires, Université d'Abomey-Calavi, Bénin; p. 52.

Puterflam J, Bouvarel I, Ragot O, Drouet M. 2007. Contamination des élevages de poulets de chair par Campylobacter: quels moyens de maîtrise? In Septièmes Journées de la Recherche Avicole, Tours, France, 521-523. 